Rapid review of cognitive screening instruments in MCI: Proposal for a process-based approach modification of overlapping tasks in select widely used instruments

\title{
Authors:
}

Unai Díaz-Orueta (School of Nursing and Human Sciences, Dublin City University, Dublin, Ireland)

Alberto Blanco-Campal (Health Service Executive, Ardee, Co. Louth, Ireland)

Teresa Burke (School of Nursing and Human Sciences, Dublin City University, Dublin, Ireland)

Corresponding author: Dr. Unai Diaz-Orueta, Dublin City University, School of Nursing and Human Sciences. Glasnevin, Dublin 9, Dublin (Ireland). Phone: +353 1700 8034. Email: unai.diaz-orueta@dcu.ie,undiaz@gmail.com

Funding: This study was funded by the European Commission, under the program MSCA-IF (Marie Sklodowska Curie Actions-Individual Fellowship), Grant Number 654895 -ESPACE-H2020-MSCA-IF-2014. 


\begin{abstract}
Background: A detailed neuropsychological assessment plays an important role in the diagnostic process of Mild Cognitive Impairment (MCI). However, available brief cognitive screening tests for this clinical population are administered and interpreted based mainly, or exclusively, on total achievement scores. This score-based approach can lead to erroneous clinical interpretations unless we also pay attention to the test taking behaviour or to the type of errors committed during test performance.

Methods: The goal of the current study is to perform a rapid review of the literature regarding cognitive screening tools for dementia in primary and secondary care; this will include revisiting previously published systematic reviews on screening tools for dementia, extensive database search, and analysis of individual references cited in selected studies.

Results: A subset of representative screening tools for dementia was identified that covers as many cognitive functions as possible. How these screening tools overlap with each other (in terms of the cognitive domains being measured and the method used to assess them) was examined and a series of process-based approach modifications for these overlapping features were proposed, so that the changes recommended in relation to one particular cognitive task could be extrapolated to other screening tools.

Conclusion: It is expected that future versions of cognitive screening tests, modified using a process-based approach, will highlight the benefits of attending to qualitative features of test performance when trying to identify subtle features suggestive of MCI and/or dementia.
\end{abstract}

\title{
Keywords
}

Dementia. Cognitive Assessment. Mild Cognitive Impairment (MCI). Screening. 


\section{Rapid review of cognitive screening instruments in MCI: Proposal for a process-based approach modification of overlapping tasks in select widely used instruments}

Multiple concepts have been proposed to capture the connection between the subclinical cognitive changes associated with age and pathological alterations in cognition (e.g. Mild Cognitive Decline, Mild Neurocognitive Decline, Cognitive Impairment-No Dementia, Mild Cognitive Impairment) (Ritchie \& Touchon, 2000). Of these, by far the most widely adopted in both research and clinical practice is that of Mild Cognitive Impairment (MCI), referring to a clinical syndrome that represents an intermediate but abnormal state of cognitive impairment between normal ageing and dementia (Petersen, Smith, Waring, Ivnik, Tangalos, \& Kokmen 1999). Initially focusing exclusively on memory impairment, considered prodromal to Alzheimer's disease, MCI is now considered a broad construct, heterogeneous in its clinical presentations, with several neuropsychological syndromes or clinical subtypes having been identified (i.e. MCI amnestic single or multiple domains and non-amnestic single and multiple domain) which are potentially due to multiple aetiologies (e.g. Alzheimer's disease, vascular disease, etc.) (Petersen et al., 2014; Winblad et al., 2004). The heterogeneous aetiology of MCI is reflected in the findings from cumulative conversion rates, which show a $30 \%$ conversion to any type of dementia, coupled with high rates of reversion to a non-clinical status (Ritchie \& Ritchie, 2012).

More recently, the Diagnostic and Statistical Manual of Mental Disorders, Fifth Edition (DSM-5), in an effort to capture the pre-dementia stage of cognitive impairments includes the term 'mild neurocognitive disorder' (mNCD). This concept is derived almost exclusively from previous research in MCI (Sachs-Ericsson \& Blazer, 2015) and its operational criteria resemble the revised Mayo Clinic criteria for MCI (Winblad et al., 2004) in that there has to be (i) concern expressed by the individual or an informant or observations 
made by the clinician; (ii) objective impairment in one or more cognitive domains, preferably documented by standardised neuropsychological testing; and (iii) preserved independence in functional abilities. Although the construct of mNCD also represents a heterogeneous category, compared to the concept of MCI it encompasses a more diverse group of entities, including mild acquired impairments in younger individuals and impairments that may be transient, static, or even reversible. The terms mNCD and MCI are not, therefore, interchangable. That being said, as we intend to focus on early detection of dementia, we will stick to the widely used term of MCI as a non-reversible condition that precedes dementia and will focus on how that detection can be improved with the resources available in a common primary care setting.

In Ireland, 4,000 new cases of dementia are detected ever year and there are currently approximately 41,470 people living with dementia, which is expected to triple by 2050 , constituting a challenge for patients and their families, professionals and society at large (Cahill, O’Shea \& Pierce, 2012). Not surprisingly, early detection of MCI and dementia and the identification of the specific underlying disease causing these syndromes in order to treat them is a public health priority. Among other medical investigations, including functional assessment, biological markers and neuroimaging techniques, a detailed objective cognitive evaluation is considered to play an important role in the diagnostic process (Albert et al., 2011).

Although objective evidence of cognitive decline is a core feature of MCI, establishing the demarcation between normal cognition and MCI and indeed between MCI and dementia is not straightforward for a number of reasons.

First, there are, as yet, no universally accepted, or indeed recommended, guidelines as to what set of cognitive tests should be used to detect MCI, although most criteria allude to the importance of examining all the main cognitive areas (Petersen et al., 2014). In this vein, 
with reference to the identification and clinical characterisation of MCI cases of the amnestic subtype (aMCI) participating in clinical trials, Stephan et al. (2013) detected a large heterogeneity in the neuropsychological methods used to determine memory impairment in different trials, together with a lack of uniformity in the clinical diagnosis of this syndrome, a problem that the authors felt extends to other clinical states such as dementia including Alzheimer's disease, Lewy Body, or frontotemporal or vascular dementia.

Second, although cognitive decline or impairment that "falls below the expected level" (DSM-5) or is "beyond that expected for both age and education level" (Petersen et al., 1999) is an essential part of the operation criteria for MCI, there is no gold standard or even widely accepted cut-off scores to operationalize this concept which is complicated by the fact that few cognitive tests have adequate norms for the oldest old (i.e. $\geq 90$ years). With relatively little empirical justification, the Mayo Clinic Criteria for MCI uses a cut off score of $1-1.5$ standard deviations below normative values although fulfilment of this criteria is ultimately determined through clinical judgement (Winblad et al., 2004), whereas the new DSM-5 criteria for mNCD suggest using a wider spectrum with performance typically lying in the 1-2 standard deviation range (between the $3^{\text {rd }}$ percentile and $16^{\text {th }}$ percentile). These discrepancies inevitably lead to considerable variation in prevalence and conversion rates (Marcos et al., 2016; Ritchie \& Ritchie, 2012; Ritchie \& Touchon, 2000).

Third, current MCI criteria establish the requirement of objective cognitive impairment in one or more cognitive domains but there is ambiguity regarding the number of impaired indices or cognitive measures needed to fulfil cognitive syndrome criteria (Petersen et al., 2014). Furthermore, establishing that a particular defective score on a given cognitive measure is the result of cognitive impairment in the cognitive domain that the test portrays to measure, may lead to spurious clinical conclusions as any cognitive test, by its very nature, is multifactorial and places demands on more than just one cognitive process. For example, so 
called visuoconstruction tasks, such as the clock drawing, rely not only on visuospatial processes but also on semantic memory and executive control (Rouleau, Salmon \& Butters, 1996).

Fourth, while an in-depth neuropsychological evaluation of a wide range of cognitive domains is considered to be optimal for the detection and clinical differentiation of MCI subtypes, access to tertiary services such as memory clinics with the full complement of neuropsychological evaluation is relatively rare and most clinical cognitive examinations are conducted using brief cognitive screening measures for different purposes in a variety of clinical settings with different levels of specialist training in administration and interpretation of cognitive test performance (Lonie et al., 2010). In a previous systematic review of 15 different cognitive screening instruments -most of which were designed to detect early and moderate stage dementia - Lonie, Tierney and Ebmeier (2009) concluded that while several of these measures afforded the clinician the ability to detect MCI, none of them wholly fulfilled all the criteria considered to be important in MCI screening. More specifically, these authors could not find data about (1) sensitivity for early atypical dementia presentations, (2) test specificity when compared with psychiatric and non-progressive neurological conditions, (3) cross-cultural usage and (4) reliability and predictive validity.

The cognitive screening tools typically used to detect MCI in clinical practice differ in terms of the number of cognitive domains they cover, some covering each of the primary cognitive domains of cognitive function, typically referred to as "comprehensive" and others providing only partial cover "non-comprehensive" (Lonie et al 2009; 2010). However, and in relation to the core of the present review, what they do have in common is that their administration and method of interpretation rely almost exclusively on a total achievement score. In fact, all of them provide a cut-off score for ease of interpretation and an impaired overall score is typically used to detect MCI in research studies and clinical practice. There 
are, however, many limitations with this standard restrictive 'quantitative method' of interpretation of cognitive test performance that, in many cases, can lead to erroneous clinical interpretations. As previously noted, an impaired score on a given test can be attributable to a range of underlying cognitive deficits, the nature of which would be hidden under a single index score.

In order to circumvent this shortcoming, in this review we propose to complement the traditional quantitative analysis of test performance in cognitive screening measures with the qualitative methodology developed by Kaplan (1988) which emphasizes the importance of the analysis of processes and errors in understanding brain-behaviour relationships. To elaborate, beyond a 'traditional' cognitive evaluation that pays attention almost exclusively to 'how much' a person achieves on a cognitive task (total test score), a more qualitative, process-based, approach that observes 'how' a task is completed (i.e. what kind of cognitive strategies are adopted) and 'why' the person fails on the task, can aid in the process of early and differential diagnosis of MCI. To illustrate, two patients, with different underlying neuropathology, may obtain exactly the same score in a cognitive test but the way in which they approach the task, as well as the specific underlying cognitive strategies that they recruit to attain the score, may be very different. Moreover, the reason for their failure in completing the task may also differ. This rich qualitative information can be invaluable for clinicians in their quest to determine the most likely underlying pathology responsible for cognitive failure.

This approach to understanding the underlying cognitive processes by means of paying attention to test taking behaviour and types of errors committed is widely known as the process-based approach (PBA), or Boston Process-Approach (BPA), to neuropsychological evaluation. According to Milberg, Hebben and Kaplan (1986), the BPA is a result of the work started by Edith Kaplan in the late "60s at the Clinical 
Neuropsychological Services at the Boston Veterans Administration Medical Center (USA), and derives from the gradual combination of tests that had been proven valid in the clinical discrimination of patients with and without brain damage with tests that purported to measure narrow specifiable cognitive functions. Kaplan and her team performed careful systematic observations of the problem-solving strategies used by patients (i.e. the way they successfully solved or failed to solve each problem presented to them). The resulting method allowed both a quantitative assessment of a patient's performance and a dynamic serial "picture" of the information-processing style that each patient used.

With the advantages of such an approach in mind, the goals of the current study are to (1) perform a rapid review of the literature regarding cognitive screening tools for MCI and dementia in primary and secondary care; this will include revisiting previously published systematic reviews on screening tools for dementia, extensive database search, and analysis of individual references cited in selected studies, (2) identify a subset of representative screening tools for dementia that cover as many cognitive functions as possible, (3) identify how these cognitive screening tools overlap with each other (in terms of the cognitive domains being measured and the method used to assess them), and (4) propose a series of process-based approach modifications for these overlapping features, so that the changes recommended in one particular cognitive task can be extrapolated to the same task as it appears in the other screening tools.

\section{Methodology}

\section{Searching strategy}

Screening tests were identified by searching electronic databases (PubMed, PsycINFO and Ingenta Connect), using combinations of the terms already used by Cullen et al. (2007) in their systematic review (“dementia”, “Alzheimer", "cognitive impairment”, "post stroke”, "screen", "primary care" and "community") in order to replicate the findings and to update 
the existing tools for the last 10 year period. The search was complemented with the combination of words "cognitive screening", "screen", "systematic review", "MCI", "Alzheimer", "dementia", and excluding words "children" and "schizophrenia", in order to address those existing systematic reviews that have been performed for the last 10 years since the work by Cullen et al. (2007). Additionally, databases were searched for the terms "Boston process approach", "qualitative error analysis" and "quantified process approach" (to ensure inclusion of the work performed by Poreh (2000) to systematise the previous work done by Kaplan and colleagues on the BPA), in order to find out not only additional tests but also procedures and variables that could have already been used in the adaptation of existing tests to a process-based approach. Individual test names were also used as search terms and the reference lists of papers yielded were manually searched for those studies identified as relevant when reviewing citations.

\section{Selection strategy}

The total number of tests identified as used in the evaluation of MCI and dementia by means of different sources was 160 , a number that was reduced to 153 after duplicated were removed. Those tests already conceived or modified using a process-based approach to cognitive evaluation $(n=48)$ were excluded from the eligibility study, as the goal was to identify tests that were not yet modified but could potentially benefit from modifications using this approach.

\section{Results}

Figure 1 shows the PRISMA Diagram about the process followed from the initial identification of potential tests modifiable using a PBA. 
A total list of 105 potential tests was identified. At this point, the following inclusion criteria were followed to determine a potential group of screening tools that could be taken into further consideration for MCI screening:

- Tests that measure a minimum of 3 of the 6 cognitive domains mentioned in the diagnosis of dementia according to the Diagnostic and Statistical Manual of mental Disorders, Fifth Edition (DSM-5), namely, complex attention, executive function, learning and memory, language, perceptual-motor function, and social cognition.

- Tests that were designed for dementia or that are widely used in dementia assessment once there is a well-established diagnosis.

- $\quad$ Tests fulfilling the Quality Assessment tool for Diagnostic Accuracy Studies (QUADAS) (Whiting et al., 2003) (see Table 1).

\section{(INSERT TABLE 1 AROUND HERE)}

- Interviews, observation scales or informant ratings, computer tasks and remotescreening instruments (for example, telephone administered tests) were excluded, as well as tests being in languages other than English.

- An additional number of tests that were unavailable, out of print, or restricted to a specific geographic area in the world, were also excluded.

The application of this selection strategy led to the exclusion of another 84 tests, thus leading to a list of potential 21 screening tests for further quality review. None of these tests had previously undergone significant changes using a process-based approach.

\section{Quality review strategy}

The quality review of these 21 tests showed that some had copyright conflicts for research use (e.g. MMSE), some were specifically designed for a particular type of dementia once the diagnosis was established (e.g. ADAS-Cog), were also excluded. One test (i.e. Cognitive Abilities Screening Instrument -CASI) was excluded based on the recommendation 
done by the main author of the test herself. Finally, tests that require high qualification levels to administer, that is, they cannot be administered by a wide range of professionals were excluded (unless they met the QUADAS criteria, as maybe with a process-based approach administration and scoring, the level of widespread use among different professionals can be extended). This criteria of potential benefits derived from a BPA were only applied to the Repeatable Battery for Neuropsychological Status (RBANS), which was included due to its widespread use and popularity among a wide range of clinicians.

The outcome of the quality review is a list of 7 screening tools in Table 2 . None of these tests has previously undergone a process-based approach, or have, at most, undergone a qualitative error analysis approach.

(INSERT TABLE 2 AROUND HERE)

\section{Data extraction: identification of cognitive tasks' overlap}

We identified the cognitive tasks that were shared, albeit with subtle variations, between these cognitive screening tools. The following overlapping features were identified between different selected tests:

1. MoCA, ACE-III, RUDAS, and STMS include the copy of a cube as a visuoconstructional task.

2. MoCA, ACE-III, STMS, SLUMS, and Brief KSCA-R include some version of clock drawing.

3. Verbal fluency is present in the MOCA (letter F), ACE-III (letter P and animals), RUDAS (animals), SLUMS (animals), and RBANS (fruits and vegetables)

4. MoCA, STMS, and Brief KSCA-r comprise a similarities subtest for abstraction.

5. MoCA, ACE-III, RUDAS, SLUMS and STMS include short-term recall of a series of 3 to 5 words, while RBANS includes a 10-word list with 4 trials for immediate memory, one for delayed free recall and one for recognition. 
6. MoCA, SLUMS and STMS include digit span, but it is only digit forward for STMS and RBANS, and only backward for SLUMS, while MoCA includes both.

\section{Proposal for modifications using a process-based approach}

In order to develop process-based approach versions of cognitive screening tasks, we drew from the work of different authors who have already shown how the analysis of errors conveys additional information regarding underlying brain/ behaviour relations (e.g., Trail Making Test or verbal fluency). We took inspiration from the previous work by Price et al. (2011), who had already indicated the benefits of the analysis of errors as produced on the

Clock Drawing Test drawing in the MoCA subtest; and the benefits of adding a copy condition to help identify the primary underlying cognitive deficits responsible for errors in the command condition. We also drew from the work of Hodges, Salmon and Butters (1991) on the error analysis of the Boston Naming Test, proving to be very effective beyond the overall achievement score in distinguishing between Huntington's disease and Alzheimer's disease. Furthermore, the analysis of errors presented for the fluency task is inspired by the work of Troyer, Moscovitch and Winocur (1997).

Informed by the methodological framework of the Process Based Approach, we employ two main methodologies (Poreh, 2000) in order to capture the underlying cognitive deficits responsible for test underperformance:

- The "Satellite" Testing Paradigm: This approach consists of the inclusion of complimentary conditions to the existing cognitive task with the aim of isolating the individual cognitive processes necessary for its completion in order to assist in the identification of the precise nature of the cognitive deficit responsible for test performance on the original cognitive task. Using this paradigm, satellite conditions for the Clock Drawing Task (e.g. copy and tracing conditions) and the Naming Task (i.e. semantic and phonetic cue) were introduced. 
- Composition Paradigm: This approach consists of the generation of new indices in already existing cognitive task, using the data that has already been derived from the standardized administration of this task but not previously analysed. New indices are derived for Verbal Fluency tests (i.e. switching and clustering) and Memory tasks (e.g. gained and lost access). A series of qualitative classifications of errors are proposed for a series of tasks including Trail Making, Clock Drawing, Verbal Fluency, and Memory.

Due to the described overlapping of some of the subtests or items included throughout different cognitive screening measures, it was possible to identify how to easily implement process-based approach modifications to particular cognitive screening tasks that may be extrapolated to all administration procedures among the different tests containing the same task, as shown below:

1. Visuoconstructional tasks (copy cube): as we stated above, MoCA, ACE-III, RUDAS, and STMS include the copy of a cube as a visuoconstructional task, though the one in STMS is flipped 180 degrees horizontally. In this case, the following indices are proposed: (1) the test respondent represents a $2 \mathrm{D}$ shape instead of the $3 \mathrm{D}$ model (which may reflect a contrast sensitivity deficit) (Cronin-Golomb, 2011) (2) drawing overlaps model (pull to stimulus): the test taker draws partially over the given model; (3) lines missing: it may reflect visuoperceptive or attentional problems (spatial positioning error); (4) motor perseveration in line drawing; (5) tremor or segmentation, and (5) rotation.

2. Clock drawing: Variations among different screening tests are very heterogeneous and obtained information depends on the type of conditions administered (command, copy, tracing). Interestingly, the BKSCA-R breaks down the tasks of writing the numbers ("I want you to write in the numbers, as on a clock face") and writing the 
hands ("and on this circle draw in the hands to make it say 9 o'clock"), followed by a clock in which only hands must be drawn to set time, and a last clock on which time must be read. After the administration of all different tests that include one or other type of clock drawing condition, it is considered of great interest to take into consideration that modifications performed to the clock drawing just with the inclusion of a copy (Price et al., 2011) and a tracing condition (Evans, Coen, Burke \& Lawlor, 2005) can be the most comprehensive approach for the evaluation of the following areas: graphomotor performance, conceptualization or time representation, spatial and/or planning abilities, and detection of perseveration / pull to stimulus.

3. Verbal fluency: according to a recent study by Vaughan, Coen, Kenny and Lawlor (2016), the inclusion of a semantic fluency task that serves as a comparison with phonemic fluency may add significant value to the screening for MCI and dementia, and can be quickly added to any assessment protocol. This study found that the semantic advantage (i.e. better performance for animal versus letter $\mathrm{F}$ fluency) persists into later life in a population-based sample of community-dwelling older adults, and that this pattern is reversed in Alzheimer's dementia (i.e. loss of semantic advantage in Alzheimer's disease, yielding a phonemic advantage). Hence, the inclusion of both types of fluency tasks and the comparison of their performance (in the form of discrepancy scores between phonemic and semantic fluency, for example) can help distinguishing between normal cognitive aging and defective cognitive aging. From our BPA perspective, based on existing literature (Troyer, 2000), it is also important to consider the following indices (1) registering answers in 15 second intervals (i.e. 0-15, 15-30, 30-45 and 45-60 seconds intervals), (2) set loss errors (i.e. errors that violate the instructions given) and (3) indexes for clustering (for measuring semantic categorisation) and switching (as a proxy for cognitive flexibility) can be 
generalized to each and every test that uses verbal fluency as a part of the cognitive assessment. According to Troyer et al. (1997), an examination of clustering and switching scores can provide information about why a particular participant performs well or poorly on these tasks, and these are sensitive to the effects of age and to conditions of divided attention.

4. Similarities: As all the MoCA, STMS, and the Brief KSCA-r comprise a similarities subtest for abstract thinking, the examples in Table 3 in relation to the error analysis included in the MoCA can be extended to the rest of the tests as well, as a way to capture subtle abstract thinking differences that may help differentiate between different neurocognitive conditions.

\section{(INSERT TABLE 3 HERE)}

5. Orientation questions: A confusion in the day of the week for one day (i.e. saying it is Thursday when it is actually Friday) and a confusion on the season of the year (mainly, between Summer and Autumn) may not show subtle differences between healthy individuals. It is yet to be seen how the administration of these types of questions can give rise to different conclusions with participants with MCI and dementia, as a wrong identification of time and space becomes a clearer symptom of underlying cognitive deficits.

6. Short-term recall of words: MOCA, ACE-III, RUDAS, SLUMS and STMS include short term recall of 3 to 5 words (depending on the test). Separately, the word recall in the Brief KSCA-r rises to 10 words and has a free recall and a recognition task, which is closer to the paradigm used in tests like the California Verbal Learning Test (CVLT) (Delis et al., 1987) or the Philadelphia (repeatable) Verbal Learning Test (P(r)VLT) (Price et al., 2004). Depending on the needs of the clinician, a decision needs to be made on the number of words, as the qualitative approach may differ 
depending on the number of items used. For lists from 3 to 5 words, a free recall followed by category cues and either phonetic or a recognition task is appropriate. However, for an in-depth assessment of memory using the P(r)VLT that includes long-term recall (as long as it may be with a 20 minute delay, instead of the 5 minute delay used in the cognitive screening measures used here), it may be necessary to include or explore other additional indices. So far, the work undertaken included the consideration of the following qualitative indices: serial order, primacy and recency effect, gained and lost access, intrusions and Perseverative errors, and repetitions.

7. For Digit Span, it was concluded that there is a need to administer both forward and backward digits as they rely on different cognitive processes domains and thus on different strategies. What needs to be considered is which series length is the most appropriate for both forward and backward digits. The original MoCA only tested a series of 4 digits forward and a series of 3 digits backwards. We introduced 2 series of 5 digits forwards and 2 series of 4 digits backwards to test the limits. However, the STMS includes series of 5, 6 and 7 digit forwards that resemble the series used in Wechsler Memory Scales. In any case, the qualitative indices that have already been developed (as in Lamar et al., 2013) may be used regardless of the length of the series. These indices are: (1) percentage of digits recalled in any order [(total number of correct digits in any order/total possible correct)*100], (2) percentage of digits recalled in serial order [(total number of correct digits in serial order/(total possible correct)*100], (3) omissions, (4) additions, (5) substitutions, and (6) capture errors (e.g. for 1-4-9-3, “3-4-9-1”; and for 7-2-8-6, “6-7-8-2”). 


\section{Conclusion: future directions in the screening of MCI and dementia}

Modification of classic cognitive instruments using a process-based approach is not new and some of the best existing examples are the Wechsler Intelligence Scales for Adults revised as a Neuropsychological Instrument (WAIS-R-NI, Kaplan et al., 1991) and the Delis-Kaplan Executive Function System (D-KEFS) (Delis et al., 2001), which mainly integrates a compilation of nine classic tests for the assessment of executive functions. For specific assessment in older populations, the Kaplan-Baycrest Neurocognitive Assessment was developed based on the principles of the process approach (Leach et al., 2000). However, for the process-based approach to neuropsychological assessment to gain popularity in the aim to improve classic versions of briefer cognitive screening tests, research is now required in order to quantify the qualitative observations derived from applying this BPA to test taking behaviours observed during cognitive screening test performance and psychometric analysis of the validity and clinical utility of test modifications is also required (Erickson, 1995). We consider that this effort is justified and we expect that future versions of cognitive screening tests modified using a BPA will highlight the benefits of paying attention to qualitative features of test performance when trying to identify subtle features suggestive of MCI and/or dementia. Additionally, it would be interesting to extend the scope of the identification of subtle features to 'cognitive frailty', defined as a particular state of cognitive vulnerability in MCI and other similar clinical entities exposed to vascular risk, with a subsequent increased progression to vascular dementia (VaD) (Ruan et al., 2017). This would, together with the consideration of features of Subjective Cognitive Decline (SCD) (understood as a state of experienced cognitive difficulties that may take place as early as 5 years before the onset of MCI (Jessen et al., 2014; Molinuevo et al., 2017; Ruan et al., 2017)), allow clinicians to administer a more accurate process-based screening to rule out different preclinical entities. 
We do, however, recognise that no cognitive screening test on its own can be considered a valid replacement for a more in-depth neuropsychological assessment.

\section{Conflicts of interest}

None. 


\section{Description of author's roles}

All the authors formulated the research question. U. Díaz-Orueta performed the literature review and wrote the initial draft of the paper. U. Díaz-Orueta and A. Blanco-Campal collaborated in writing and developing the review results. T. Burke performed final edits. 


\section{References}

Albert, M.S., DeKosky, S.T., Dickson, D., Dubois, B., Feldman, H.H., Fox, N.C, ...,

Phelps, C.H. (2011). The diagnosis of mild cognitive impairment due to Alzheimer's disease: recommendations from the National Institute on Aging-Alzheimer's Association workgroups on diagnostic guidelines for Alzheimer's disease. Alzheimer's \& Dementia: The Journal of the Alzheimer's Association, 7(3), 270-279.

Cahill, S., O'Shea, E., \& Pierce, M. (2012). Creating Excellence in Dementia Care. A Research Review for Ireland's National Dementia Strategy. Dublin: Trinity College.

Cronin-Golomb, A. (2011). Visuospatial Function in Alzheimer's Disease and Related Disorders. In A.E. Budson, \& N.W. Kowall (Eds.). The Handbook of Alzheimer's Disease and Other Dementias, First Edition (pp. 457-482). London: Blackwell.

Cullen, B., O’Neill, B., Evans, J.J., Coen, R.F., \& Lawlor, B.A. (2007). A review of screening tests for cognitive impairment. Journal of Neurology, Neurosurgery, and Psychiatry, 78(8), 790-799.

Delis, D.C., Kaplan, E., \& Kramer, J.H. (2001). Delis-Kaplan Executive Function System (D-KEFS). San Antonio, TX: The Psychological Corporation.

Delis, D.C., Kramer, J.H., Kaplan, E., \& Ober, B.A. (1987). California Verbal Learning Test: Adult version. Manual. San Antonio, TX: Psychological Corporation.

Erickson, R.C. (1995). A review and critique of the process approach in neuropsychological assessment. Neuropsychology Review, 5(4), 223-243.

Evans, I., Coen, R., Burke, T., \& Lawlor, B.A. (2005). Clock drawing and executive function in Alzheimer's disease. Conference Paper. Irish Journal of Medical Science, 174(3, Supp.2), 80-80.

Hodges, J.R., Salmon, D.P., \& Butters, N. (1991). The nature of the naming deficit in Alzheimer's and Huntington's disease. Brain, 114(4), 1547-1558. 
Hopkins, R., Kilik, L., Day, D., Rows, C., \& Hamilton, P. (2005). The Brief Kingston Standardized Cognitive Assessment -Revised. International Journal of Geriatric Psychiatry, 20, 227-231.

Hsieh, S., Schubert, S., Hoon, C., Mioshi, E., \& Hodges, J.R. (2013). Validation of the Addenbrooke's Cognitive Examination III in Frontotemporal Dementia and Alzheimer's Disease. Dementia and Geriatric Cognitive Disorders, 36, 242.

Kokmen, E., Naessens, J.M., \& Offord, K.P. (1987). A short test of mental status: description and preliminary results. Mayo Clinic Proceedings, 62, 281-288.

Jessen, F., Amariglio, R.E., van Boxtel, M., Breteler, M., Ceccaldi, M., Chételat, G., Dubois, B., ..., Wagner, M., Subjective Cognitive Decline Initiative (SCD-I) Working group. (2014). A conceptual framework for research on subjective cognitive decline in preclinical Alzheimer's disease. Alzheimer's \& Dementia, 10(6), 844-852.

Kaplan, E. (1988). A process approach to neuropsychological assessment. In T. Boll \& B.K. Bryant (Eds.). Clinical neuropsychology and brain function: research, measurement, and practice (pp. 129-167). Washington, DC: American Psychological Association.

Kaplan, E., Fein, D., Morris, R, \& Delis, D.C. (1991). WAIS-R as a Neuropsychological Instrument. Toronto, Canada: The Psychological Corporation, Harcourt Brace Jovanovich.

Lamar, M., Rhodes, E., Wambach, D.M., Swenson, R., \& Libon, D.J. (2013). Digit Span as a neuropsychological instrument of error processing. In L. Ashendorf, R. Swenson, \& D. Libon (2013). The Boston Process Approach to Neuropsychological Assessment: A Practitioner's Guide (pp. 65-76). New York: Oxford University Press.

Leach, L., Kaplan, E., Rewilak, D., \& Proulx, G.B. (2000). Kaplan Baycrest Neurocognitive Assessment (KBNA). San Antonio, TX: Pearson Education. 
Lonie, J.A., Parra, M., Tierney, K.M., Herrmann, L.L., Donaghey, C., O'Carroll, R.E. \& Ebmeier, K.P. (2010). Predicting outcome in mild cognitive impairment: 4-year follow-up study. The British Journal of Psychiatry, 197(2), 135-140.

Lonie, J.A., Tierney, K.M., \& Ebmeier, K.P. (2009). Screening for mild cognitive impairment : a systematic review. International Journal of Geriatric Psychiatry, 24(9), 902-915.

Marcos, G., Santabárbara, J., Lopez-Anton, R., De-la-Cámara, C., Gracia-García, P., Lobo, E., ..., Lobo, A. (2016). Conversion to dementia in mild cognitive impairment diagnosed with DSM-5 criteria and with Petersen's criteria. Acta Psychiatrica Scandinava, 133(5), 378-385.

Milberg, W.P., Hebben, N.A., \& Kaplan, E. (1986). The Boston process approach to neuropsychological assessment". In G. Adams (Ed.). Neuropsychological Assessment of Neuropsychiatric Disorders (pp. 42-65). New York: Oxford University Press.

Molinuevo, J.L., Rabin, L.A., Amariglio, R., Buckley, R., Dubois, B., Ellis, K.A., Ewers, M., ..., Frank Jessen, for the Subjective Cognitive Decline Initiative (SCD-I) Working Group (2017). Implementation of subjective cognitive decline criteria in research studies. Alzheimer's \& Dementia, 13(3), 296-311.

Nasreddine, Z.S., Phillips, N.A., Bedirian, V., Charbonneau, S., Whitehead, V., Collin, I., ... Chertkow, H. (2005). The Montreal Cognitive Assessment, MoCA: A Brief Screening. Journal of the American Geriatrics Society, 53, 695-699.

Petersen, R.C., Smith, G.E., Waring, S.C., Ivnik, R.J., Tangalos, E.G., \& Kokmen, E. (1999). Mild cognitive impairment: clinical characterization and outcome. Archives of Neurology, 56, 303-308. 
Petersen, R.C., Caracciolo, B., Brayne, C., Gauthier, S., Jelic, V., \& Fratiglioni, L. (2014). Mild Cognitive Impairment: A concept in evolution. Journal of Internal Medicine, 275, 214-228.

Poreh, A. (2000). The quantified process approach: an emerging methodology to neuropsychological assessment. The Clinical Neuropsychologist, 14(2), 212-222.

Price, C.C., Cunningham, H., Coronado, N., Freedland, A., Cosentino, S., Penney, D.L., ..., Libon, D.J. (2011). Clock Drawing in the Montreal Cognitive Assessment: Recommendations for Dementia Assessment. Dementia and Geriatric Cognitive Disorders, 31(3), 179-187.

Price, C.C., Garrett, K.D., Libon, D.J., Swenson, R., Penney, D., \& Jefferson, A., et al. (2004). Verbal serial list learning among dementia patients with and without white matter changes: Factor solutions.Journal of the International Neuropsychological Society, 10, S1-S8.

Randolph, C., Tierney, M.C., Mohr, E., \& Chase, T.N. (1998). The Repeatable Battery for the Assessment of Neuropsychological Status (RBANS): preliminary clinical validity. Journal of Clinical and Experimental Neuropsychology, 20(3), 310-319.

Ritchie, K., \& Ritchie, C. (2012). Mild Cognitive Impairment (MCI) twenty years on. International Psychogeriatrics, 24(1), 1-5.

Ritchie, K, \& Touchon, J. (2000). Mild Cognitive Impairment: conceptual basis and current nosological status. Lancet, 355, 225-228.

Rouleau, I., Salmon, D., \& Butters, N. (1996). Longitudinal analysis of clock drawing in Alzheimer's disease patients. Brain and Cognition, 31, 17-34.

Ruan, Q., D’Onofrio, G.D., Sancarlo, D., Greco, A., Lozupone, M., Seripa, D., Panza, F., \& Yu, Z. (2017). Emerging biomarkers and screening for cognitive frailty. Aging Clinical and Experimental Research. doi: 10.1007/s40520-017-0741-8. 
Sachs-Ericsson, N. \& Blazer, D.G. (2015). The new DSM-5 diagnosis of mild neurocognitive disorder and its relation to research in mild cognitive impairment. Aging \& Mental Health, 19(1), 2-12.

Stephan, B.C., Minett, T., Pagett, E., Siervo, M., Brayne, C., \& McKeith, I.G. (2013). Diagnosing Mild Cognitive Impairment (MCI) in clinical trials: a systematic review. BMJ Open, 3(2), 1-8. http://doi.org/10.1136/bmjopen-2012-001909

Storey, J.E., Rowland, J.T.J., Basic, D., Conforti, D.A, \& Dickson, H.G. (2004). The Rowland Universal Dementia Assessment Scale (RUDAS): a multicultural cognitive assessment scale. International Psychogeriatrics /IPA, 16(1), 13-31.

Tariq, S.H., Tumosa, N., Chibnall, J.T., Perry III, H.M., \& Morley, J.E. (2006). The Saint Louis University Mental Status (SLUMS) Examination for detecting mild cognitive impairment and dementia is more sensitive than the MiniMental Status Examination (MMSE) - A pilot study. American Journal of Geriatric Psychiatry, 14, 900-910.

Troyer, A.K. (2000). Normative data for clustering and switching on verbal fluency task. Journal of Clinical and Experimental Neuropsychology, 22(3), 370-378.

Troyer, A.K., Moscovitch, M., \& Winocur, G. (1997). Clustering and switching as two components of verbal fluency: evidence from younger and older healthy adults. Neuropsychology, 11(1), 138-146.

Vaughan, R.M., Coen, R.F., Kenny, R.A., \& Lawlor, B.A. (2016). Preservation of the semantic verbal fluency advantage in a large population-based sample: normative data from the TILDA study. Journal of the International Neuropsychological Society, 22(5), 570-576. 
Whiting, P., Rutjes, A.W.S., Reitsma, J.B., Bossuy, P.M., \& Kleijnen, K. (2003). The development of QUADAS: a tool for the quality assessment of studies of diagnostic accuracy included in systematic reviews. BMC Medical Research Methodology, 3, 25.

Winblad, B., Palmer, K., Kivipelto, M., Jelic, V., Fratiglioni, L., Wahlund, L.O., ..., Petersen, R.C. (2004). Mild cognitive impairment--beyond controversies, towards a consensus: report of the International Working Group on Mild Cognitive Impairment. Journal of Internal Medicine, 256(3), 240-246. 
Table 1. The QUADAS Checklist (Adapted from Whiting et al., 2003)

Question to consider (Possible answers: Yes, No, Unclear)

1. Was the spectrum of patients representative of the patients who will receive the test in practice?

2. Were selection criteria clearly described?

3. Is the reference standard likely to correctly classify the target condition?

4. Is the time period between reference standard and index test short enough to be reasonably sure that the target condition did not change between the two tests?

5. Did the whole sample or a random selection of the sample, receive verification using a reference standard of diagnosis?

6. Did patients receive the same reference standard regardless of the index test result?

7. Was the reference standard independent of the index test (i.e. the index test did not form part of the reference standard)?

8. Was the execution of the index test described in sufficient detail to permit replication of the test?

9. Was the execution of the reference standard described in sufficient detail to permit its replication?

10. Were the index test results interpreted without knowledge of the results of the reference standard?

11. Were the reference standard results interpreted without knowledge of the results of the index test?

12. Were the same clinical data available when test results were interpreted as would be available when the test is used in practice?

13. Were uninterpretable/ intermediate test results reported?

14. Were withdrawals from the study explained? 
Table 2. Screening tools that may potentially benefit from a process-based approach

\begin{tabular}{|c|c|c|c|c|}
\hline Test Name & Acronym & $\begin{array}{l}\text { Author } \\
\text { (Year) }\end{array}$ & Domains Assessed & $\begin{array}{c}\text { Administration Time } \\
\text { (Minutes) }\end{array}$ \\
\hline $\begin{array}{l}\text { Montreal Cognitive } \\
\text { Assessment }\end{array}$ & $\begin{array}{l}\text { MoCA (full } \\
\text { version and } \\
\text { short version) }\end{array}$ & $\begin{array}{l}\text { Nasreddine } \\
\text { et al. (2005) }\end{array}$ & $\begin{array}{l}\text { Short term memory, } \\
\text { visuospatial abilities, } \\
\text { cognitive flexibility, } \\
\text { attention, } \\
\text { concentration, working } \\
\text { memory, language, } \\
\text { verbal fluency, time } \\
\text { and spatial orientation }\end{array}$ & $10-15$ \\
\hline $\begin{array}{l}\text { Addenbrooke's } \\
\text { Cognitive } \\
\text { Examination -Third } \\
\text { Edition }\end{array}$ & ACE-III & $\begin{array}{l}\text { Hsieh et al. } \\
(2013)\end{array}$ & $\begin{array}{l}\text { Orientation, } \\
\text { registration, attention } \\
\text { and concentration, } \\
\text { memory (recall, } \\
\text { anterograde memory, } \\
\text { retrograde memory), } \\
\text { verbal fluency (p, } \\
\text { animals), language } \\
\text { (comprehension, } \\
\text { writing, repetition, } \\
\text { naming, reading), } \\
\text { visuospatial abilities, } \\
\text { perceptual abilities, } \\
\text { recall, recognition }\end{array}$ & 20 \\
\hline $\begin{array}{l}\text { (Brief) Kingston } \\
\text { Standardized } \\
\text { Cognitive } \\
\text { Assessment -Revised }\end{array}$ & BKSCA-R & $\begin{array}{l}\text { Hopkins et } \\
\text { al. }(2005)\end{array}$ & $\begin{array}{l}\text { Orientation, word } \\
\text { recall, abstract } \\
\text { thinking, spatial } \\
\text { inversion, clock } \\
\text { drawing, motor } \\
\text { perseveration, word } \\
\text { delayed recall, word } \\
\text { recognition }\end{array}$ & 30 \\
\hline $\begin{array}{l}\text { Rowland Universal } \\
\text { Dementia } \\
\text { Assessment Scale }\end{array}$ & RUDAS & $\begin{array}{l}\text { Storey et al. } \\
(2004)\end{array}$ & $\begin{array}{l}\text { memory, language, } \\
\text { visual context, visual- } \\
\text { spatial }\end{array}$ & $6-10$ \\
\hline $\begin{array}{l}\text { Sant Louis } \\
\text { University Mental } \\
\text { Status Exam } \\
\end{array}$ & SLUMS & $\begin{array}{l}\text { Tariq et al. } \\
(2006)\end{array}$ & $\begin{array}{l}\text { memory, language, } \\
\text { visual context, visual- } \\
\text { spatial }\end{array}$ & $6-10$ \\
\hline $\begin{array}{l}\text { Short Test of Mental } \\
\text { Status }\end{array}$ & STMS & $\begin{array}{l}\text { Kokmen, } \\
\text { Naessens, \& } \\
\text { Offord } \\
(1987)\end{array}$ & $\begin{array}{l}\text { Attention, immediate } \\
\text { memory, calculus, } \\
\text { abstraction, copy and } \\
\text { construction, } \\
\text { information, recall. }\end{array}$ & 5 \\
\hline $\begin{array}{l}\text { Repeatable Battery } \\
\text { for } \\
\text { Neuropsychological } \\
\text { Status }\end{array}$ & RBANS & $\begin{array}{l}\text { Randolph et } \\
\text { al. (1998) }\end{array}$ & $\begin{array}{l}\text { Immediate memory, } \\
\text { visuospational and } \\
\text { visuoconstructional, } \\
\text { attention, language, } \\
\text { delayed memory }\end{array}$ & 30 \\
\hline
\end{tabular}


Table 3. Error analysis in similarities subtest (examples for the MoCA)

\begin{tabular}{|c|c|c|c|}
\hline Error & Error Type & Description & Example \\
\hline \multicolumn{4}{|l|}{ Category } \\
\hline \multirow{9}{*}{ In-Set } & Vague & Superordinate, but & Train-Bicycle: "they go fast" \\
\hline & & superficial categorical & Watch-Ruler: "you can use \\
\hline & & response & them" \\
\hline & Subordinate & Response that relates to & Train-Bicycle: "both have \\
\hline & & shared concrete attributes & wheels" \\
\hline & & or to highly specific & Watch-Ruler: "both have \\
\hline & & properties about the test & numbers" \\
\hline & & items that may not be & \\
\hline & & correct in all instances & \\
\hline \multirow{12}{*}{ Out-of-Set } & One Object & Response that pertains to & Train-Bicycle: "one is the like \\
\hline & & only one member of the & other minus the engine" \\
\hline & & word pair & Watch-Ruler: "the watch tells \\
\hline & & & the time" \\
\hline & Juxtaposition & Description of how one & Train-Bicycle: "the bicycle can \\
\hline & & member of the word pair & go inside the train" \\
\hline & & might interact with the & Watch-Ruler: "the ruler can \\
\hline & & other member & measure the watch" \\
\hline & Different & Description of how the & Train-Bicycle: "one has a motor \\
\hline & & items of the word pair are & and the other one has not" \\
\hline & & different & Watch-Ruler: "one is round and \\
\hline & & & the other one is rectangular" \\
\hline
\end{tabular}


Figure 1. PRISMA Flow Diagram for the identification and selection of screening tests for early detection Mild Cognitive Impairment potentiallly modifiable using a processbased approach (PBA)

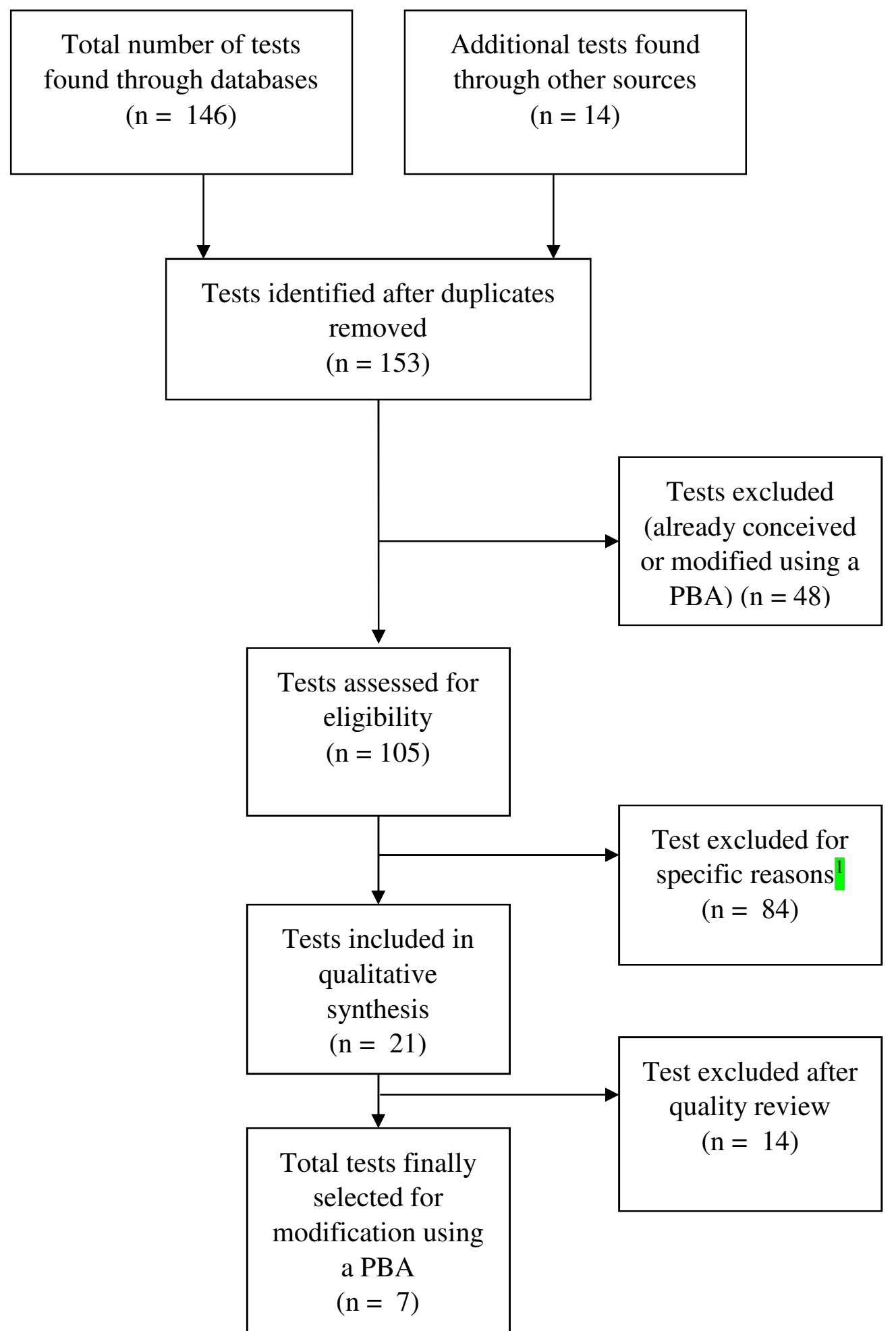

${ }^{1}$ Reasons: the test was excluded for measuring less than 3 relevant cognitive functions $(n=51)$; the test itself or further information about the test could not be located $(n=13)$; the test was informant-based $(n=9)$; the test was for later stages of a well-established diagnosis $(n=7)$; the test was administered on the phone $(n=2)$; the test was not in English $(\mathrm{n}=2)$. 